\title{
OTR10 Proposta de um sistema integrado de identificação e investigação de microorganismos no Controle de Qualidade em Indústrias Farmacêuticas
}

Luciane Martins Medeiros ${ }^{1}$, Cledir Santos², Nelson Lima², Manuela da Silva $^{3}$, Verônica Viana Vieira ${ }^{4}$

${ }^{1}$ Bio-Manguinhos, Fiocruz, Rio de Janeiro, RJ

2 Centro de Engenharia Biológica, Universidade do Minho

${ }^{3}$ VPPLR, Presidência, Fiocruz, Rio de Janeiro, RJ

${ }^{4}$ Instituto Oswaldo Cruz, Fiocruz, Rio de Janeiro, RJ

Introdução: A microbiota em ambientes industriais farmacêuticos apresenta grande diversidade, nem sempre tendo sua identificação concluída pelo Controle de Qualidade (CQ). Métodos estão sendo propostos para superar este problema, como espectrometria de massa por MALDI-TOF e métodos genotípicos. Outro problema consiste na investigação de fontes de contaminação, realizada apenas por registros impressos. A proposta de uma abordagem holística, que abranja a identificação da microbiota autóctone em nível de espécie e a investigação fidedigna das fontes de contaminação, se mostra como uma necessidade do $\mathrm{CQ}$.

Objetivo: Propor um sistema integrado de identificação de microorganismos e de investigação de fontes de contaminação que consiga atender as necessidades do $\mathrm{CQ}$

Metodologia: Foi elaborado um fluxograma do sistema proposto e, para verificar sua funcionalidade, bactérias isoladas na rotina do $\mathrm{CQ}$ foram submetidas à triagem de identificação proposta. Os isolados foram criopreservados $\left(-80^{\circ} \mathrm{C}\right)$ na Coleção de Culturas de Microorganismos Autóctones (CCMA) e seus dados foram inseridos em um banco de dados (BD). Uma amostragem dos isolados não identificados na triagem $(\mathrm{N}=36)$ foi submetida ao kit MicroSEQ 500 e submetida ao MALDI-TOF, para a construção de um BD "in house". Para avaliar a interface entre o armazenamento de cepas e de informações numa investigação de fonte de contaminação, foram selecionados 11 isolados da CCMA correlacionados à ocorrência de um desvio. Para esta avaliação, foi utilizada a técnica de eletroforese de gel em campo pulsado (PFGE).

Resultados: O sistema proposto reduziu o tempo e o custo de identificação pelo $\mathrm{CQ}$ de Bio-Manguinhos: redução em $30 \%$ do custo por amostra e de $24 \mathrm{~h}$ para $20 \mathrm{~min}$ o tempo de resultado. $\mathrm{Na}$ identificação, a porcentagem dos não identificados também diminuiu, quando comparada ao método anteriormente utilizado. Todas as 36 bactérias foram identificadas pelo MicroSEQ e tiveram seus perfis proteômicos inseridos no BD do MALDI-TOF. Destas, 8 cepas foram identificadas como Micrococcus, sendo $4 \mathrm{M}$. luteus. Estes últimos 
foram utilizados para a construção de um espectro de referência em MALDI-TOF, que foi introduzido no BD "in house". Na investigação de um evento de contaminação, foi possível a recuperação das cepas envolvidas pelo uso do BD da CCMA. Na análise por PFGE destas cepas foi evidenciada a provável fonte de contaminação.

Conclusão: $O$ espectro de referência criado a partir de cepas autóctones de $M$. luteus permitiu a ampliação do BD do MALDI-TOF com sucesso e outras cepas não identificadas estão sendo introduzidas, seguindo o mesmo procedimento. A CCMA foi útil na recuperação de cepas envolvidas em desvios da qualidade e permitiu a investigação de sua origem de contaminação pela técnica de PFGE. O sistema proposto se mostrou viável, reduziu custos e apresentou melhorias na identificação de cepas e na investigação de fontes de contaminação. Apoio financeiro: CNPq (CsF) e FIOTEC.

Palavras-Chave: Identificação Microbiana, Controle de Qualidade, MALDI-TOF, Coleções 